\title{
Inequalities for Dual Orlicz Mixed Quermassintegrals
}

\section{Lijuan Liu}

School of Mathematics and Computational Science, Hunan University of Science and Technology, Xiangtan, China

Email: lijuanliu@hnust.edu.cn

How to cite this paper: Liu, L.J. (2016) Inequalities for Dual Orlicz Mixed Quermassintegrals. Advances in Pure Mathematics, 6, 894-902.

http://dx.doi.org/10.4236/apm.2016.612067

Received: October 14, 2016

Accepted: November 14, 2016

Published: November 17, 2016

Copyright $\odot 2016$ by author and Scientific Research Publishing Inc. This work is licensed under the Creative Commons Attribution International License (CC BY 4.0).

http://creativecommons.org/licenses/by/4.0/

\begin{abstract}
In this paper, we establish the dual Orlicz-Minkowski inequality and the dual OrliczBrunn-Minkowski inequality for dual Orlicz mixed quermassintegrals.
\end{abstract}

\section{Keywords}

Star Body, Orlicz Radial Sum, Dual Orlicz Mixed Volume

\section{Introduction}

Recently, Convex Geometry Analysis has made great achievement in Orlicz space (see [1]-[14]). Zhu, Zhou and Xu [12] defined the Orlicz radial sum and dual Orlicz mixed volumes. Let $\mathcal{C}^{+}$be the set of convex and strictly decreasing functions $\phi:(0,+\infty) \rightarrow(0,+\infty)$ such that $\lim _{t \rightarrow \infty} \phi(t)=0, \lim _{t \rightarrow 0} \phi(t)=\infty$ and $\phi(0)=\infty$.

Let $K$ and $L$ be two star bodies about the origin in $\mathbb{R}^{n}$ and $a, b \geq 0$; the Orlicz radial sum $a \cdot K \tilde{+}_{\phi} b \cdot L$ was defined by [13]

$$
\rho_{a \cdot K \tilde{\phi}_{\phi} b \cdot L}(u)=\sup \left\{t>0: a \phi\left(\frac{\rho_{K}(u)}{t}\right)+b \phi\left(\frac{\rho_{L}(u)}{t}\right) \leq \phi(1)\right\}, \forall u \in S^{n-1} .
$$

The case $\phi(t)=t^{-p}(p \geq 1)$ of the Orlicz radial sum is the $L_{p}$ harmonic radial sum, which was defined by Lutwak (see [15]).

Let $f_{r}^{\prime}$ denote the right derivative of a real-valued function $f$. For $\phi \in \mathcal{C}^{+}$, there is $\phi_{r}^{\prime}(1)<0$ because $\phi$ is convex and strictly decreasing. The dual Orlicz mixed volume $\tilde{V}_{\phi}(K, L)$ is defined by

$$
\frac{n}{\phi_{r}^{\prime}(1)} \tilde{V}_{\phi}(K, L)=\lim _{\varepsilon \rightarrow 0^{+}} \frac{V\left(K \tilde{+}_{\phi} \varepsilon \cdot L\right)-V(K)}{\varepsilon} .
$$

In this paper, we will define the dual Orlicz mixed quermassintegral 


$$
\begin{aligned}
& \tilde{W}_{\phi, i}(K, L)(i=0, \cdots, n-1) \text { by } \\
& \quad \frac{n-i}{\phi_{r}^{\prime}(1)} \tilde{W}_{\phi, i}(K, L)=\lim _{\varepsilon \rightarrow 0^{+}} \frac{\tilde{W}_{i}\left(K \tilde{+}_{\phi} \varepsilon \cdot L\right)-\tilde{W}_{i}(K)}{\varepsilon} .
\end{aligned}
$$

The main purpose of this paper is to establish the dual Orlicz-Minkowski inequality and the dual Orlicz-Brunn-Minkowski inequality for dual Orlicz mixed quermassintegrals.

Theorem 1.1 Let $K$ and $L$ be two star bodies about the origin in $\mathbb{R}^{n}$ and $\phi \in \mathcal{C}^{+}$. If $0 \leq i<n-1$, then

$$
\tilde{W}_{\phi, i}(K, L) \geq \tilde{W}_{i}(K) \phi\left(\left(\frac{\tilde{W}_{i}(L)}{\tilde{W}_{i}(K)}\right)^{\frac{1}{n-i}}\right),
$$

with equality if and only if $K$ and $L$ are dilates of each other.

Theorem 1.2 Let $K$ and $L$ be two star bodies about the origin in $\mathbb{R}^{n}$ and $\phi \in \mathcal{C}^{+}$. If $0 \leq i<n-1$, then

$$
\phi(1) \geq \phi\left(\left(\frac{\tilde{W}_{i}(K)}{\tilde{W}_{i}\left(K \tilde{+}_{\phi} L\right)}\right)^{\frac{1}{n-i}}\right)+\phi\left(\left(\frac{\tilde{W}_{i}(L)}{\tilde{W}_{i}\left(K \tilde{+}_{\phi} L\right)}\right)^{\frac{1}{n-i}}\right),
$$

with equality if and only if $K$ and $L$ are dilates of each other.

This paper is organized as follows: In Section 2 we introduce above interrelated notations and their background materials. Section 3 contains the proofs of our main results.

\section{Notation and Background Material}

The radial function $\rho_{K}(u): S^{n-1} \rightarrow[0, \infty)$ of a compact star-shaped about the origin $K \in \mathbb{R}^{n}$ is defined, for $u \in S^{n-1}$, by

$$
\rho_{K}(u)=\max \{\lambda \geq 0: \lambda u \in K\} .
$$

If $\rho_{K}(\cdot)$ is positive and continuous, then $K$ is called a star body about the origin. The set of star bodies about the origin in $\mathbb{R}^{n}$ is denoted by $\mathcal{S}_{0}^{n}$. Obviously, for $K, L \in \mathcal{S}_{0}^{n}$,

$$
K \subseteq L \Leftrightarrow \rho_{K}(u) \leq \rho_{L}(u), \forall u \in S^{n-1}
$$

If $\frac{\rho_{K}(u)}{\rho_{L}(u)}$ is independent of $u \in S^{n-1}$, then we say star bodies $K$ and $L$ are dilates of each other.

If $K_{i} \in \mathcal{S}_{0}^{n}(i=1,2, \cdots, m)$ and $\lambda_{i}(i=1,2, \cdots, m)$ are nonnegative real numbers, then the volume of $\lambda_{1} K_{1} \tilde{+} \cdots \tilde{+} \lambda_{m} K_{m}$ is a homogeneous polynomial of degree $n$ in $\lambda_{i}$ given by

$$
V\left(\lambda_{1} K_{1} \tilde{+} \cdots \tilde{+} \lambda_{m} K_{m}\right)=\sum_{i_{1}, \cdots, i_{n}} \tilde{V}\left(K_{i_{1}}, \cdots, K_{i_{n}}\right) \lambda_{i_{1}} \cdots \lambda_{i_{n}},
$$

where the sum is taken over all $n$-tuples $\left(i_{1}, \cdots, i_{n}\right)$ of positive integers not exceeding $m$. 
The coefficient $\tilde{V}\left(K_{i_{1}}, \cdots, K_{i_{n}}\right)$ depends only on the bodies $K_{i_{1}}, \cdots, K_{i_{n}}$, and is uniquely determined by the above identity, it is called the dual mixed volume of $K_{i_{1}}, \cdots, K_{i_{n}}$. More explicitly, the dual mixed volume $\tilde{V}\left(K_{i_{1}}, \cdots, K_{i_{n}}\right)$ has the following integral representation [16]:

$$
\tilde{V}\left(K_{i_{1}}, \cdots, K_{i_{n}}\right)=\frac{1}{n} \int_{S^{n-1}} \rho_{K_{i_{1}}}(u) \cdots \rho_{K_{i_{n}}}(u) \mathrm{d} S(u),
$$

where $S$ is the Lebesgue measure on $S^{n-1}$.

The coefficients $\tilde{V}\left(K_{i_{1}}, \cdots, K_{i_{n}}\right)$ are nonnegative, symmetric and monotone (with respect to set inclusion). They are also multilinear with respect to the radial sum and $\tilde{V}(K, \cdots, K)=V(K)$. Let $K_{1}=\cdots=K_{n-i}=K$ and $K_{n-i+1}=\cdots=K_{n}=L$, then the dual mixed volume $\tilde{V}\left(K_{1}, \cdots, K_{n}\right)$ is usually written as $\tilde{V}_{i}(K, L)$. If $L=B$, then $\tilde{V}_{i}(K, B)$ is the dual quermassintegral $\tilde{W}_{i}(K)$. For $0 \leq i \leq n-1$, the dual mixed quermassintegral $\tilde{W}_{i}(K, L)$ denotes the dual mixed volume $\tilde{V}(\underbrace{K, \cdots, K}_{n-i-1}, \underbrace{B, \cdots, B}_{i}, L)$. For $L=K$, then $\tilde{W}_{i}(K, L)=\tilde{W}_{i}(K)$.

The dual mixed quermassintegral $\tilde{W}_{i}(K, L)$ has the following integral representation:

$$
\tilde{W}_{i}(K, L)=\frac{1}{n} \int_{S^{n-1}} \rho_{K}^{n-i-1}(u) \rho_{L}(u) \mathrm{d} S(u)
$$

where $S$ is the Lebesgue measure on $S^{n-1}$.

By using the Minkowski's integral inequality, we can obtain the dual Minkowski inequality for dual mixed quermassintegrals: If $K, L \in \mathcal{S}_{0}^{n}$, and $0 \leq i<n-1$, then

$$
\tilde{W}_{i}(K, L)^{n-i} \leq \tilde{W}_{i}(K)^{n-i-1} \tilde{W}_{i}(L),
$$

equality holds if and only if $K$ and $L$ are dilates of each other.

Suppose that $\mu$ is a probability measure on a space $X$ and $g: X \rightarrow I \subset \mathbb{R}$ is a $\mu$ intergrable function, where $I$ is a possibly infinite interval. Jessen's inequality states that if $\phi: X \rightarrow I \subset \mathbb{R}$ is a convex function, then

$$
\int_{X} \phi(g(x)) \mathrm{d} \mu(x) \geq \phi\left(\int_{X} g(x) \mathrm{d} \mu(x)\right) .
$$

If $\phi$ is strictly convex, equality holds if and only if $g(x)$ is a constant for $\mu$-almost all $x \in X \quad$ (see [17]).

\section{Main Results}

Let $K, L \in \mathcal{S}_{0}^{n}$ and $\phi \in \mathcal{C}^{+}$. For $i=0, \cdots, n-1$, the dual Orlicz mixed quermassintegral $\tilde{W}_{\phi, i}(K, L)$ is defined by

$$
\tilde{W}_{\phi, i}(K, L)=\frac{1}{n} \int_{S^{n-1}} \phi\left(\frac{\rho_{L}(u)}{\rho_{K}(u)}\right) \rho_{K}^{n-i}(u) \mathrm{d} S(u) .
$$

For $L=K$, then $\tilde{W}_{\phi, i}(K, K)=\phi(1) \tilde{W}_{i}(K)$. The case $i=0$ of the dual Orlicz mixed quermassintegral $\tilde{W}_{\phi, i}(K, L)$ is the dual Orlicz mixed volume $\tilde{V}_{\phi}(K, L)$, which was defined by Zhu, Zhou and $\mathrm{Xu}[12]$. 
Corollary 3.1 The dual Orlicz mixed quermassintegral $\tilde{W}_{\phi, i}(K, \cdot)$ is monotone with respect to set inclusion.

Proof. Let $L_{1}, L_{2} \in \mathcal{S}_{0}^{n}$ and $L_{1} \subseteq L_{2}$. By (3.1), (2.2) and the fact that $\phi$ is strictly decreasing on $(0, \infty)$, we have

$$
\begin{aligned}
\tilde{W}_{\phi, i}\left(K, L_{1}\right) & =\frac{1}{n} \int_{S^{n-1}} \phi\left(\frac{\rho_{L_{1}}(u)}{\rho_{K}(u)}\right) \rho_{K}^{n-i}(u) \mathrm{d} S(u) \\
& \geq \frac{1}{n} \int_{S^{n-1}} \phi\left(\frac{\rho_{L_{2}}(u)}{\rho_{K}(u)}\right) \rho_{K}^{n-i}(u) \mathrm{d} S(u) \\
& =\tilde{W}_{\phi, i}\left(K, L_{2}\right) .
\end{aligned}
$$

Lemma 3.1 [12] Let $K, L \in \mathcal{S}_{0}^{n}$ and $u \in S^{n-1}$. If $\phi \in \mathcal{C}^{+}$, then

$$
a \phi\left(\frac{\rho_{K}(u)}{t}\right)+b \phi\left(\frac{\rho_{L}(u)}{t}\right)=\phi(1)
$$

if and only if

$$
\rho_{a \cdot K \tilde{F}_{\phi} b \cdot L}(u)=t .
$$

Lemma 3.2 [12] Let $K, L \in \mathcal{S}_{0}^{n}$ and $\phi \in \mathcal{C}^{+}$. Then

$$
\lim _{\varepsilon \rightarrow 0^{+}} \frac{\rho_{\tilde{r}_{\phi} \varepsilon \cdot L}(u)-\rho_{K}(u)}{\varepsilon}=\frac{\rho_{K}(u)}{\phi_{r}^{\prime}(1)} \phi\left(\frac{\rho_{L}(u)}{\rho_{K}(u)}\right),
$$

uniformly for all $u \in S^{n-1}$.

Theorem 3.1 Let $K, L \in \mathcal{S}_{0}^{n}$ and $\phi \in \mathcal{C}^{+}$. For $i=0, \cdots, n-1$, then

$$
\lim _{\varepsilon \rightarrow 0^{+}} \frac{\tilde{W}_{i}\left(K \tilde{+}_{\phi} \varepsilon \cdot L\right)-\tilde{W}_{i}(K)}{\varepsilon}=\frac{n-i}{n \phi_{r}^{\prime}(1)} \int_{s^{n-1}} \phi\left(\frac{\rho_{L}(u)}{\rho_{K}(u)}\right) \rho_{K}^{n-i}(u) \mathrm{d} S(u) .
$$

Proof. Suppose $\varepsilon>0, K, L \in \mathcal{S}_{0}^{n}$, and $u \in S^{n-1}$. Note that $K \tilde{+}_{\phi} \varepsilon \cdot L \rightarrow K$ as $\varepsilon \rightarrow 0^{+}$(see [12]). By Lemma 3.2, it follows that

$$
\begin{aligned}
\lim _{\varepsilon \rightarrow 0^{+}} \frac{\rho_{K \tilde{F}_{\phi} \varepsilon \cdot L}^{n-i}(u)-\rho_{K}^{n-i}(u)}{\varepsilon} & =\left.(n-i) \rho_{K \tilde{f}_{\phi} \varepsilon \cdot L}^{n-i-1}(u)\right|_{\varepsilon=0} \cdot \lim _{\varepsilon \rightarrow 0^{+}} \frac{\rho_{K \tilde{f}_{\phi} \varepsilon \cdot L}(u)-\rho_{K}(u)}{\varepsilon} \\
& =\frac{(n-i) \rho_{K}^{n-i}(u)}{\phi_{r}^{\prime}(1)} \phi\left(\frac{\rho_{L}(u)}{\rho_{K}(u)}\right),
\end{aligned}
$$

uniformly on $S^{n-1}$.

Hence

$$
\begin{aligned}
\lim _{\varepsilon \rightarrow 0^{+}} \frac{\tilde{W}_{i}\left(K \tilde{+}_{\phi} \varepsilon \cdot L\right)-\tilde{W}_{i}(K)}{\varepsilon} & =\lim _{\varepsilon \rightarrow 0^{+}}\left(\frac{1}{n} \int_{S^{n-1}} \frac{\rho_{K \tilde{x}_{\phi} \varepsilon \cdot L}^{n-i}(u)-\rho_{K}^{n-i}(u)}{\varepsilon} \mathrm{d} S(u)\right) \\
& =\frac{1}{n} \int_{S^{n-1}} \lim _{\varepsilon \rightarrow 0^{+}} \frac{\rho_{K \tilde{f}_{\phi} \varepsilon \cdot L}^{n-i}(u)-\rho_{K}^{n-i}(u)}{\varepsilon} \mathrm{d} S(u) \\
& =\frac{n-i}{n \phi_{r}^{\prime}(1)} \int_{S^{n-1}} \phi\left(\frac{\rho_{L}(u)}{\rho_{K}(u)}\right) \rho_{K}^{n-i}(u) \mathrm{d} S(u) .
\end{aligned}
$$


We complete the proof of Theorem 3.1.

From (3.1) and Theorem 3.1, we have

$$
\frac{n-i}{\phi_{r}^{\prime}(1)} \tilde{W}_{\phi, i}(K, L)=\lim _{\varepsilon \rightarrow 0^{+}} \frac{\tilde{W}_{i}\left(K \tilde{+}_{\phi} \varepsilon \cdot L\right)-\tilde{W}_{i}(K)}{\varepsilon} .
$$

For $K \in \mathcal{S}_{0}^{n}$, since $\frac{1}{n} \int_{S^{n-1}} \rho_{K}^{n-i}(u) \mathrm{d} S(u)=\tilde{W}_{i}(K)$, then $\frac{\rho_{K}^{n-i}(\cdot) \mathrm{d} S(\cdot)}{n \tilde{W}_{i}(K)}$ is a probability measure on $S^{n-1}$.

\section{Proof of Theorem 1.1}

By (3.1), (2.6), (2.5) and the fact that $\phi$ is decreasing on $(0, \infty)$, we obtain

$$
\begin{aligned}
\frac{\tilde{W}_{\phi, i}(K, L)}{\tilde{W}_{i}(K)} & =\frac{1}{n \tilde{W}_{i}(K)} \int_{S^{n-1}} \phi\left(\frac{\rho_{L}(u)}{\rho_{K}(u)}\right) \rho_{K}^{n-i}(u) \mathrm{d} S(u) \\
& \geq \phi\left(\frac{1}{n \tilde{W}_{i}(K)} \int_{S^{n-1}} \frac{\rho_{L}(u)}{\rho_{K}(u)} \rho_{K}^{n-i}(u) \mathrm{d} S(u)\right) \\
& =\phi\left(\frac{\tilde{W}_{i}(K, L)}{\tilde{W}_{i}(K)}\right) \\
& \geq \phi\left(\frac{\tilde{W}_{i}(K)^{\frac{n-i-1}{n-i}} \tilde{W}_{i}(L)^{\frac{1}{n-i}}}{\tilde{W}_{i}(K)}\right) \\
& =\phi\left(\left(\frac{\tilde{W}_{i}(L)}{\tilde{W}_{i}(K)}\right)^{\frac{1}{n-i}}\right)
\end{aligned}
$$

This gives the desired inequality. Since $\phi$ is strictly decreasing, from the equality condition of the dual Minkowski inequality (2.5), we have that $K$ and $L$ are dilates of each other.

Conversely, when $L=\lambda K$, by (3.1), we have

$$
\tilde{W}_{\phi, i}(K, L)=\tilde{W}_{i}(K) \phi(\lambda)=\tilde{W}_{i}(K) \phi\left(\left(\frac{\tilde{W}_{i}(L)}{\tilde{W}_{i}(K)}\right)^{\frac{1}{n-i}}\right) .
$$

The following uniqueness is a direct consequence of the dual Orlicz-Minkowski inequality (1.4).

Corollary 3.2 Suppose $\phi \in \mathcal{C}^{+}$, and $\mathcal{M} \subset \mathcal{S}_{0}^{n}$ such that $K, L \in \mathcal{M}$. For $0 \leq i<n-1$, if

$$
\tilde{W}_{\phi, i}(M, K)=\tilde{W}_{\phi, i}(M, L) \text {, for all } M \in \mathcal{M} \text {, }
$$

or

$$
\frac{\tilde{W}_{\phi, i}(K, M)}{\tilde{W}_{i}(K)}=\frac{\tilde{W}_{\phi, i}(L, M)}{\tilde{W}_{i}(L)}, \text { for all } M \in \mathcal{M},
$$

then $K=L$. 
Proof. Suppose (3.4) holds. If we take $K$ for $M$, then from (3.1), we obtain

$$
\phi(1) \tilde{W}_{i}(K)=\tilde{W}_{\phi, i}(K, K)=\tilde{W}_{\phi, i}(K, L) .
$$

Hence, from the dual Orlicz-Minkowski inequality (1.4), we have

$$
\phi(1) \geq \phi\left(\left(\frac{\tilde{W}_{i}(L)}{\tilde{W}_{i}(K)}\right)^{\frac{1}{n-i}}\right)
$$

with equality if and only if $K$ and $L$ are dilates of each other. Since $\phi$ is strictly decreasing on $(0, \infty)$, we have

$$
\tilde{W}_{i}(L) \geq \tilde{W}_{i}(K)
$$

with equality if and only if $K$ and $L$ are dilates of each other. If we take $L$ for $M$, we similarly have $\tilde{W}_{i}(L) \leq \tilde{W}_{i}(K)$. Hence, $\tilde{W}_{i}(K)=\tilde{W}_{i}(L)$ and from the equality condition we can conclude that $K$ and $L$ are dilates of each other. However, since they have the same volume they must be equal.

Next, suppose (3.5) holds. If we take $K$ for $M$, then from (3.1), we obtain

$$
\phi(1)=\frac{\tilde{W}_{\phi, i}(K, K)}{W_{i}(K)}=\frac{\tilde{W}_{\phi, i}(L, K)}{\tilde{W}_{i}(L)} .
$$

Then, from the dual Orlicz-Minkowski inequality (1.4), we have

$$
\phi(1) \geq \phi\left(\left(\frac{\tilde{W}_{i}(K)}{\tilde{W}_{i}(L)}\right)^{\frac{1}{n-i}}\right),
$$

with equality if and only if $K$ and $L$ are dilates of each other. Since $\phi$ is strictly decreasing on $(0, \infty)$, we have

$$
\tilde{W}_{i}(K) \geq \tilde{W}_{i}(L)
$$

with equality if and only if $K$ and $L$ are dilates of each other. If we take $L$ for $M$, we similarly have $\tilde{W}_{i}(K) \leq \tilde{W}_{i}(L)$. Hence, $\tilde{W}_{i}(K)=\tilde{W}_{i}(L)$ and from the equality condition we can conclude that $K$ and $L$ are dilates of each other. However, since they have the same volume they must be equal.

From the dual Orlicz-Minkowski inequality, we will prove the following dual Orlicz-Brunn-Minkowski inequality which is more general than Theorem 1.2.

Theorem 3.2 Let $K, L \in \mathcal{S}_{0}^{n}, a, b>0$ and $\phi \in \mathcal{C}^{+}$. If $0 \leq i<n-1$, then

$$
\phi(1) \geq a \phi\left(\left(\frac{\tilde{W}_{i}(K)}{\tilde{W}_{i}\left(a \cdot K \tilde{+}_{\phi} b \cdot L\right)}\right)^{\frac{1}{n-i}}\right)+b \phi\left(\left(\frac{\tilde{W}_{i}(L)}{\tilde{W}_{i}\left(a \cdot K \tilde{+}_{\phi} b \cdot L\right)}\right)^{\frac{1}{n-i}}\right),
$$

with equality if and only if $K$ and $L$ are dilates of each other.

Proof. Let $K_{\phi}=a \cdot K \tilde{+}_{\phi} b \cdot L$. From (2.3), Lemma 3.1 and (1.4), it follows that 


$$
\begin{aligned}
\phi(1) & =\frac{1}{n \tilde{W}_{i}\left(K_{\phi}\right)} \int_{s^{n-1}} \phi(1) \rho_{K_{\phi}}^{n-i}(u) \mathrm{d} S(u) \\
& =\frac{1}{n \tilde{W}_{i}\left(K_{\phi}\right)} \int_{s^{n-1}}\left[a \phi\left(\frac{\rho_{K}(u)}{\rho_{K_{\phi}}(u)}\right)+b \phi\left(\frac{\rho_{L}(u)}{\rho_{K_{\phi}}(u)}\right)\right] \rho_{K_{\phi}}^{n-i}(u) \mathrm{d} S(u) \\
& =\frac{a}{n \tilde{W}_{i}\left(K_{\phi}\right)} \int_{s^{n-1}} \phi\left(\frac{\rho_{K}(u)}{\rho_{K_{\phi}}(u)}\right) \rho_{K_{\phi}}^{n}(u) \mathrm{d} S(u)+\frac{b}{n \tilde{W}_{i}\left(K_{\phi}\right)} \int_{S^{n-1}} \phi\left(\frac{\rho_{L}(u)}{\rho_{K_{\phi}}(u)}\right) \rho_{K_{\phi}}^{n-i}(u) \mathrm{d} S(u) \\
& =\frac{a}{\tilde{W}_{i}\left(K_{\phi}\right)} \tilde{W}_{\phi, i}\left(K_{\phi}, K\right)+\frac{b}{\tilde{W}_{i}\left(K_{\phi}\right)} \tilde{W}_{\phi, i}\left(K_{\phi}, L\right) \\
& \geq a \phi\left(\left(\frac{\tilde{W}_{i}(K)}{\tilde{W}_{i}\left(K_{\phi}\right)}\right)^{\frac{1}{n-i}}\right)+b \phi\left(\left(\frac{\tilde{W}_{i}(L)}{\tilde{W}_{i}\left(K_{\phi}\right)}\right)^{\frac{1}{n-i}}\right) .
\end{aligned}
$$

By the equality condition of the dual Orlicz-Minkowski inequality (1.4), equality in (3.6) holds if and only if $K$ and $L$ are dilates of each other.

Indeed, we also can prove the dual Orilcz-Minkowski inequality by the dual OrilczBrunn-Minkowski inequality.

Proof. For $\varepsilon \geq 0$, let $K_{\varepsilon}=K \tilde{+}_{\phi} \varepsilon \cdot L$. Note that $K_{\varepsilon} \rightarrow K$ as $\varepsilon \rightarrow 0^{+}$. By the dual Orlicz-Brunn-Minkowski inequality, the following function

$$
G(\varepsilon)=\phi\left(\left(\frac{\tilde{W}_{i}(K)}{\tilde{W}_{i}\left(K_{\varepsilon}\right)}\right)^{\frac{1}{n-i}}\right)+\varepsilon \phi\left(\left(\frac{\tilde{W}_{i}(L)}{\tilde{W}_{i}\left(K_{\varepsilon}\right)}\right)^{\frac{1}{n-i}}\right)-\phi(1)
$$

is non-positive. Obviously, $G(0)=0$. Thus

$$
\lim _{\varepsilon \rightarrow 0^{+}} \frac{G(\varepsilon)-G(0)}{\varepsilon} \leq 0
$$

On the other hand, we have

$$
\begin{aligned}
& \lim _{\varepsilon \rightarrow 0^{+}} \frac{G(\varepsilon)-G(0)}{\varepsilon}=\lim _{\varepsilon \rightarrow 0^{+}} \frac{\left.\phi\left(\frac{\tilde{W}_{i}(K)}{\tilde{W}_{i}\left(K_{\varepsilon}\right)}\right)^{\frac{1}{n-i}}\right)+\varepsilon \phi\left(\left(\frac{\tilde{W}_{i}(L)}{\tilde{W}_{i}\left(K_{\varepsilon}\right)}\right)^{\frac{1}{n-i}}\right)-\phi(1)}{\varepsilon} \\
& =\lim _{\varepsilon \rightarrow 0^{+}} \frac{\phi\left(\left(\frac{\tilde{W}_{i}(K)}{\tilde{W}_{i}\left(K_{\varepsilon}\right)}\right)^{\frac{1}{n-i}}\right)-\phi(1)}{\varepsilon}+\phi\left(\left(\frac{\tilde{W}_{i}(L)}{\tilde{W}_{i}(K)}\right)^{\frac{1}{n-i}}\right) \\
& =\lim _{\varepsilon \rightarrow 0^{+}} \frac{\left.\phi\left(\frac{\tilde{W}_{i}(K)}{\tilde{W}_{i}\left(K_{\varepsilon}\right)}\right)^{\frac{1}{n-i}}\right)-\phi(1)}{\left(\frac{\tilde{W}_{i}(K)}{\tilde{W}_{i}\left(K_{\varepsilon}\right)}\right)^{\frac{1}{n-i}}-1} \cdot \lim _{\varepsilon \rightarrow 0^{+}} \frac{\left(\frac{\tilde{W}_{i}(K)}{\tilde{W}_{i}\left(K_{\varepsilon}\right)}\right)^{\frac{1}{n-i}}-1}{\varepsilon}+\phi\left(\left(\frac{\tilde{W}_{i}(L)}{\tilde{W}_{i}(K)}\right)^{\frac{1}{n-i}}\right) .
\end{aligned}
$$


Let $s=\left(\frac{\tilde{W}_{i}(K)}{\tilde{W}_{i}\left(K_{\varepsilon}\right)}\right)^{\frac{1}{n-i}}$ and note that $s \rightarrow 1^{+}$as $\varepsilon \rightarrow 0^{+}$. Consequently,

$$
\lim _{\varepsilon \rightarrow 0^{+}} \frac{\phi\left(\left(\frac{\tilde{W}_{i}(K)}{\tilde{W}_{i}\left(K_{\varepsilon}\right)}\right)^{\frac{1}{n-i}}\right)-\phi(1)}{\left(\frac{\tilde{W}_{i}(K)}{\tilde{W}_{i}\left(K_{\varepsilon}\right)}\right)^{\frac{1}{n-i}}-1}=\lim _{s \rightarrow 1^{+}} \frac{\phi(s)-\phi(1)}{s-1}=\phi_{r}^{\prime}(1) .
$$

By (3.3), we have

$$
\begin{aligned}
& \lim _{\varepsilon \rightarrow 0^{+}} \frac{\left(\left(\frac{\tilde{W}_{i}(K)}{\tilde{W}_{i}\left(K_{\varepsilon}\right)}\right)^{\frac{1}{n-i}}-1\right)}{\varepsilon} \\
= & -\lim _{\varepsilon \rightarrow 0^{+}} \frac{\tilde{W}_{i}\left(K_{\varepsilon}\right)^{\frac{1}{n-i}}-\tilde{W}_{i}(K)^{\frac{1}{n-i}}}{\varepsilon} \cdot \lim _{\varepsilon \rightarrow 0^{+}} \tilde{W}_{i}\left(K_{\varepsilon}\right)^{-\frac{1}{n-i}} \\
= & -\frac{1}{n-i} \tilde{W}_{i}(K)^{\frac{1}{n-i}-1} \cdot \lim _{\varepsilon \rightarrow 0^{+}} \frac{\tilde{W}_{i}\left(K_{\varepsilon}\right)-\tilde{W}_{i}(K)}{\varepsilon} \cdot \tilde{W}_{i}(K)^{-\frac{1}{n-i}} \\
= & -\frac{1}{\phi_{r}^{\prime}(1)} \frac{\tilde{W}_{\phi, i}(K, L)}{\tilde{W}_{i}(K)} .
\end{aligned}
$$

From (3.8), (3.9), and $(3,10)$, it follows that

$$
\lim _{\varepsilon \rightarrow 0^{+}} \frac{G(\varepsilon)-G(0)}{\varepsilon}=-\frac{\tilde{W}_{\phi, i}(K, L)}{\tilde{W}_{i}(K)}+\phi\left(\left(\frac{\tilde{W}_{i}(L)}{\tilde{W}_{i}(K)}\right)^{\frac{1}{n-i}}\right) .
$$

Combing (3.7) and (3.11), we have

$$
-\frac{\tilde{W}_{\phi, i}(K, L)}{\tilde{W}_{i}(K)}+\phi\left(\left(\frac{\tilde{W}_{i}(L)}{\tilde{W}_{i}(K)}\right)^{\frac{1}{n-i}}\right) \leq 0 .
$$

Therefore, the equality in (3.12) holds if and only if $G(\varepsilon)=G(0)=0$, this implies that $K$ and $L$ are dilates of each other.

Remark 3.1 The case $i=0$ of Theorem 1.1 and Theorem 1.2 were established by $\mathrm{Zhu}, \mathrm{Zhou}$ and $\mathrm{Xu}$ [12]. The dual forms of Theorem 1.1 and Theorem 1.2 were established by Xiong and Zou [11].

\section{References}

[1] Chen, F., Zhou, J. and Yang, C. (2011) On the Reverse Orlicz Busemann-Petty Centroid Inequality. Advances in Applied Mathematics, 47, 820-828.

http://dx.doi.org/10.1016/j.aam.2011.04.002

[2] Gardner, R.J., Hu, D. and Weil, W. (2014) The Orlicz-Brunn-Minkowski Theory: A General Framework, Additions, and Inequalities. Journal of Differential Geometry, 97, 427-476.

[3] Gardner, R.J., Hug, D., Weil, W. and Ye, D. (2015) The Dual Orlicz-Brunn-Minkowski 
Theory. Journal of Mathematical Analysis and Applications, 430, 810-829. http://dx.doi.org/10.1016/j.jmaa.2015.05.016

[4] Haberl, C., Lutwak, E., Yang, D. and Zhang, G. (2010) The Even Orlicz Minkowski Problem. Advances in Mathematics, 224, 2485-2510. http://dx.doi.org/10.1016/j.aim.2010.02.006

[5] Huang, Q. and He, B. (2012) On the Orlicz Minkowski Problem for Polytopes. Discrete \& Computational Geometry, 48, 281-297. http://dx.doi.org/10.1007/s00454-012-9434-4

[6] Li, A. and Leng, G. (2011) A New Proof of the Orlicz Busemann-Petty Centroid Inequality. Proceedings of the American Mathematical Society, 139, 1473-1481. http://dx.doi.org/10.1090/S0002-9939-2010-10651-2

[7] Ludwig, M. (2010) General Affine Surface Areas. Advances in Mathematics, 224, 23462360. http://dx.doi.org/10.1016/j.aim.2010.02.004

[8] Lutwak, E., Yang, D. and Zhang, G. (2010) Orlicz Projection Bodies. Advances in Mathematics, 223, 220-242. http://dx.doi.org/10.1016/j.aim.2009.08.002

[9] Lutwak, E., Yang, D. and Zhang, G. (2010) Orlicz Centroid Bodies. Journal of Differential Geometry, 84, 365-387.

[10] Xi, D., Jin, H. and Leng, G. (2014) The Orlicz-Brunn-Minkowski Inequality. Advances in Mathematics, 260, 350-374. http://dx.doi.org/10.1016/j.aim.2014.02.036

[11] Xiong, G. and Zou, D. (2014) Orlicz Mixed Quermassintegrals. Science China Mathematics, 57, 2549-2562. http://dx.doi.org/10.1007/s11425-014-4812-4

[12] Zhu, B., Zhou, J. and Xu, W. (2014) Dual Orlicz-Brunn-Minkowski Theory. Advances in Mathematics, 264, 700-725. http://dx.doi.org/10.1016/j.aim.2014.07.019

[13] Zhu, G. (2012) The Orlicz Centroid Inequality for Star Bodies. Advances in Applied Mathematics, 48, 432-445. http://dx.doi.org/10.1016/j.aam.2011.11.001

[14] Zou, D. and Xiong, G. (2014) Orlicz-John Ellipsoids. Advances in Mathematics, 265, 132168. http://dx.doi.org/10.1016/j.aim.2014.07.034

[15] Lutwak, E. (1996) The Brunn-Minkowski-Firey Theory II: Affine and Geominimal Surface Areas. Advances in Mathematics, 118, 244-294. http://dx.doi.org/10.1006/aima.1996.0022

[16] Lutwak, E. (1975) Dual Mixed Volumes. Pacific Journal of Mathematics, 58, 531-538. http://dx.doi.org/10.2140/pjm.1975.58.531

[17] Hardy, G.H., Littlewood, J.E. and PO'lya, G. (1988) Inequalities. 2nd Edition, Cambridge University Press, Cambridge. 
Submit or recommend next manuscript to SCIRP and we will provide best service for you:

Accepting pre-submission inquiries through Email, Facebook, LinkedIn, Twitter, etc.

A wide selection of journals (inclusive of 9 subjects, more than 200 journals)

Providing 24-hour high-quality service

User-friendly online submission system

Fair and swift peer-review system

Efficient typesetting and proofreading procedure

Display of the result of downloads and visits, as well as the number of cited articles

Maximum dissemination of your research work

Submit your manuscript at: http://papersubmission.scirp.org/

Or contact apm@scirp.org 